\title{
PARTIAL DIFFERENTIAL EQUATION FOR HEAT CONDUCTION AND ITS SOLVABILITY
}

This paper deals with the heat conduction equation which is a model of thermomechanical processes in the special case. The considered case is based on the nonstandard type of coefficients in this equation.

\section{Introduction and notations}

Let $\Omega$ be a physical body which is from the material point of view some bounded domain in the Euclidean space E3. Let $\partial \Omega$ be its surface, i.e. the boundary of the domain of $\Omega$. Let this boundary have the lipschitz piecewise smooth property. As an example we can take surfaces of polyhedrons, cones and other usual geometric configurations. Suppose this body to be nonhomogenous and nonanisotropic. Its heat conduction is in a relation to the external and internal influence sources, e.g. chemical reactions, electrical resistances etc. and to the structure of a material of the body $\Omega$. Let us know these properties in any point $x=\left(x_{1}, x_{2}, x_{3}\right)$ of $\Omega$ defined by the following functions

$$
a_{i}=a_{i}\left(x, u_{0}, u_{1}, u_{2}, u_{3}\right), i=0,1,2,3 \text {. }
$$

These functions depend on the location of a point $x$ in $\Omega$, on the searched function

$$
u_{0}=u(x, t)
$$

and on its derivatives

$$
u_{i}=\frac{\partial u(x, t)}{\partial x_{i}}, i=1,2,3 .
$$

Suppose that the function $f(x, t)$ describes an existence and actions of interior heat sources in our body $\Omega$. The dependence of the coefficients $a_{i}$ on the function $u=u_{0}$ follows from the well known fact that the heat conduction depends on the heat (see e.g. [1]). Then the equation for the heat conduction has the general form

$$
\frac{d u}{d t}-\sum_{i=1}^{3} \frac{\partial}{\partial x_{i}} a_{i}\left(x, u_{0}, u_{1}, u_{2}, u_{3}\right)-a_{0}\left(x, u_{0}, u_{1}, u_{2}, u_{3}\right)=f(x, t)
$$

For $a_{i}=\frac{\lambda}{c \rho} u_{i i}, i=1,2,3$, we have the following equation

$$
\frac{d u}{d t}=\frac{\lambda}{c \rho}\left(u_{11},+u_{22}+u_{33}\right)=f .
$$

i. e. the classical heat conduction equation for a 3-dimensional body in the form

$$
\frac{d u}{d t}-\frac{\lambda}{c \rho} \Delta u=f,
$$

where $c, \rho, \lambda$ are specific constants depending on the properties of a material and $\Delta$ is the Laplace operator (for using such operator see e.g. $[5,6])$.

A nonstationary heat conduction depends on the other input data, i.e. for our differential equations (2) or (3) from physical point of view it is necessary to suppose some boundary conditions and initial conditions. The boundary conditions characterize the heat exchange of $\Omega$ with its neighbourhood in any point of boundary $\partial \Omega$. The initial condition in the starting point of time $t_{0}=0$ describes the initial statement of heat in the body $\Omega$. Let us have the boundary conditions for $\mathrm{x} \in \partial \Omega$ and for $\mathrm{t}>0$ in the form

$$
u(x, t)=g_{1}(x, t)
$$

and

$$
\frac{d u}{d \vec{n}}(x, t)=g_{2}(x, t),
$$

where $\vec{n}$ is the normal vector to the boundary $\partial \Omega$. In the time $t_{0}=0$ we have the initial condition on $\Omega$ represented by the function

$$
u(x, 0)=h(x) .
$$

Classical problems of this type are simply solved by Fourier methods and by orthogonal function series (see e.g. [2, 5]).

\footnotetext{
* Ondrej Kovacik, Pavol Orsansky

Department of Mathematics, Faculty of Science, University of Zilina, Slovakia, E-mail: Ondrej.Kovacik@fpv.uniza.sk
} 


\section{Coefficients of heat conduction equation in nonstandard spaces}

In the paper [4] there are introduced new function spaces denoted by $L^{p(x)}$ and $W^{k, p(x)}$. The theory of these spaces is inspired by the problems of mathematical physics. The basic problem is in solvability of stationary problems of heat equations in nonstandard space, but we continue this study by dynamical problems of heat equation in nonstandard environment.

Suppose that the functions mentioned above have the growth of the type of variable powers in the variables $u_{i}, i=0,1,2,3$ Suppose that there is given some function $p(x)$ and there exist real numbers $p_{1}$ and $p_{2}$ for which

$$
1<p_{1} \leq p(x) \leq p_{2}<\infty
$$

almost everywhere on $\Omega$. The equality

$$
q(x)=\frac{p(x)}{p(x)-1}
$$

defines the so called conjugate function $q(x)$ for the function $p(x)$ on $\Omega$.

Now we assume that for the coefficients $a_{i}$ the below estimation holds

$$
\left|a_{i}\left(x, u_{0}, u_{1}, u_{2}\right)\right| \leq a_{i}(x)+\sum_{j=1}^{3} c_{i j}\left|u_{j}\right|^{p(x)-1}, i=1,2,3 .
$$

Here $c_{i j}$ are some nonnegative constants and $a_{i}(x) \geq 0$ are the functions integrable in the power $q(x)$ over $\Omega$ for $i=1,2,3$. That means that

$$
\int_{\Omega}\left|a_{i}(x)\right|^{q(x)} d x=\iiint_{\Omega}\left|a_{i}\left(x_{1}, x_{2}, x_{3}\right)\right|^{q\left(x_{1}, x_{2}, x_{3}\right)} d x_{1} d x_{2} d x_{3}<\infty .
$$

For example, we can assume that

$$
a_{i}\left(x, u_{0}, u_{1}, u_{2}, u_{3}\right)=\left|u_{i}\right|^{p(x)-1}, i=0,1,2,3 .
$$

The function $p(x)$ here we assume to be piecewise constant on $\Omega$.

For the other example we can assume that the function $p(x)$ is defined on two parts of $\Omega$, i.e. $\Omega_{1}$ and $\Omega_{2}$. In the first part for the function $p(x)$ we assume $p(x)=2$ and on the second part there is a function $p(x)=3$. Then, the relation (7) obtains the form

$$
a_{i}\left(x, u_{0}, u_{1}, u_{2}, u_{3}\right)=\left\{\begin{array}{c}
\left|u_{i}\right|, \quad x \in \Omega_{1} \\
\left|u_{i}\right|^{2}, \quad x \in \Omega_{2}=\Omega \backslash \Omega_{1}
\end{array}, i=0,1,2,3 .\right.
$$

Now we note that the theory of linear parabolic equations in the first part and on the other side in the second part of $\Omega$ is discussed in many papers and books with the problem of crossing conditions on boundary of domains $\Omega_{1}$ and $\Omega_{2}$. Our problem is defined in a very global situation. That means that we can investigate some another problems using the methods of time discretisation, so called Rothe's method.

\section{Rothe's method}

This method is based on discretisation in the time. In every moment of our time we can find the solution for stationary partial differential equation. This method is based on the decomposition of some time interval $\langle 0, T\rangle$ by the time sequence in the form $0 \leq t_{0}<t_{1} \ldots<t_{n} \leq T$ for every available natural $n$. In this time we can reformulate some property from the paper [4].

Suppose that the function $p(x)$ is defined on $\Omega$ and satisfies the conditions mentioned above. Let the functions (coefficients) $a_{i}\left(x, u_{0}, u_{1}, u_{2}, u_{3}\right)$ fulfill the next inequalities

$$
\sum_{i=0}^{3}\left[a_{i}\left(x, u_{0}, u_{1}, u_{2}, u_{3}\right)-a_{i}\left(x, v_{0}, v_{1}, v_{2}, v_{3}\right)\right]\left[u_{i}-v_{i}\right] \geq 0
$$

and

$$
\sum_{i=0}^{3}\left[a_{i}\left(x, u_{0}, u_{1}, u_{2}, u_{3}\right)\right] u_{i} \geq c_{1} \sum_{i=1}^{3}\left|u_{i}\right|^{p(x)-1}-c_{2}
$$

for any $u=\left(u_{0}, u_{1}, u_{2}, u_{3}\right), v=\left(v_{0}, v_{1}, v_{2}, v_{3}\right)$

Here we assume that these functions are defined for every $x$ from some domain $\Omega$ in $\boldsymbol{R}^{4}$ and for some positive constants $c_{1}, c_{2}$. Suppose that the first inequality is strict for all $u \neq v$. Let the boundary conditions (4) and (5) and the initial condition (6) be fulfilled. Without loss of generality we can assume that $g_{1}=g_{2}=$ $=0$ For nonhomogenous conditions on the boundary of $\Omega$ we can transform this problem into the problem with homogenous conditions.

Define a function operator

$$
H(x)=-\sum_{i=1}^{3} \frac{\partial}{\partial x_{i}} a_{i}\left(x, u_{0}, u_{1}, u_{2}, u_{3}\right)-a_{0}\left(x, u_{0}, u_{1}, u_{2}, u_{3}\right)
$$

and assume that the functions $u_{i}, H, f, \frac{\partial f}{\partial t}$ are integrable in the sense presented above on the set $\Omega$. Then, there exists a unique essentially bounded weak solution $u(x, t)$ of our problem. This solution is the classical (strictly) solution if the functions $a_{i}, f, H$, $g_{1}, g_{2}$ are at least continuous and differentiable on the product set in the form $\Omega \times\langle 0, T\rangle$.

The mentioned integration is in the Lebesgue sense, where the set of zero measure is not essential. Therefore, we call the solution to be "weak", which is more general than the classical solution.

The mentioned Rothe's method consists in some decomposition of the time interval $\langle 0, T\rangle$ with the sufficiently large numbers into small intervals with the step $T / n$. Then we have this decomposition in the form

$$
t_{0}=0<t_{1}=\frac{T}{n}<t_{2}=\frac{2 T}{n}<\dot{\mathrm{E}}<t_{n}=T .
$$

Now we use the approximation of the type

$$
\frac{d u}{d t}=\frac{u\left(x, t_{i}\right)-u\left(x, t_{i-1}\right)}{T / n}, \mathrm{t} \in\left\langle t_{i-1}, t_{i}\right\rangle, i=1,2, \ldots, n
$$


Then we have the equation in the discrete form

$$
-\left[a\left(x, u\left(x, t_{i}\right), u^{\prime}\left(x, t_{i}\right)\right]^{\prime}+u\left(x, t_{i}\right) \frac{n}{T}=f\left(x, t_{i}\right)+u\left(x, t_{i-1}\right) \frac{n}{T},\right.
$$

which is, in principle, an elliptic partial differential equation for some fixed $t_{i}$. This is based on the value of $u\left(x, t_{i-1}\right)$ which is the known function on $\Omega$, because we can go by the step by step principle from the point $t_{0}=0$ and this is an initial condition for this equation.

Therefore, we can use so called Galerkin's method with respect to the finite elements method to solve an elliptic boundary problem. Then we obtain for the time moment $t_{i}$ the solution $u\left(x, t_{i}\right)$ of the corresponding elliptic problem. In this way we obtain a set of the functions $\left\{u\left(x, t_{i}\right)\right\}, i=1,2, \ldots, n$ which are defined on the set $\Omega$. Finally, we take the following function

$$
u *(x, t)=u\left(x, t_{i-1}\right)+\frac{t-t_{i-1}}{T / n}\left[u\left(x, t_{i}\right)-u\left(x, t_{i-1}\right)\right] .
$$

Here we get the linear approximation of the solution of our initial and boundary problem for $i \in\left\langle t_{i-1}, t_{i}\right\rangle, i=1,2, \ldots, n$ of the form $u^{*}(x, t) \approx u\left(x, t_{i}\right)$. That means that we approximately know the solution for more complicated bodies in the sense of heat distribution. For more information on numerical methods the reader can refer to $[1,5,7,8,9]$.

Acknowledgement: This research has been supported by the Slovak Grant Agency VEGA through the projects No. 1/0867/08 and No. 2/0097/08.

\section{References}

[1] BARTAK, J. et al.: Partial Differential Equations - part II. (in Czech), SNTL Praha, 1988.

[2] GULDAN, V., MARCOKOVA, M.: Orthogonal Polynomials and Related Special Functions Applied in Geosciences and Technical Computations (submitted).

[3] KACUR, J.: Method of Rothe in Evolution Equations, Teubner, Leipzig 1985.

[4] KOVACIK, O., RAKOSNIK, J.: On the Spaces $L^{p(x)}$ and $W^{k, p(x)}$, Czechoslovak Math. J., 41(116) 1991, 592-618.

[5] MARCOKOVA, M., GULDAN, V.: On One Orthogonal Transform Applied on a System of Orthogonal Polynomials in Two Variables, J. Appl. Math., 2/2009, 239-245.

[6] PERICHTA, P., AC, V., ORSANSKY, P.: The Method for Calculation of the Thermomechanical Deformation, Proc. of Int. Conf Appl. Phys.Condensed Mater., 2007, 263-266.

[7] REKTORYS, K. et al.: Review of Applied Mathematics (in Czech), Academia Praha, 1995.

[8] REKTORYS, K.: Method of Time Discretization and Partial Differential Equations (in Czech), SNTL, Praha 1985.

[9] VITASEK, E.: Numerical Methods (in Czech), SNTL Praha, 1987. 